\title{
Development of Acrylic Cherenkov Counter in the KOTO experiment
}

\author{
Satoshi SHINOHARA*, for the KOTO collaboration \\ Kyoto University \\ E-mail: Shinoharaescphys.kyoto-u.ac.jp
}

The KOTO experiment aims to observe the CP-violating rare decay, $K_{L} \rightarrow \pi^{0} v \bar{v}$. This decay mode is sensitive to new physics beyond the Standard Model. For the detection of particles escaping in the beam region, an in-beam photon counter was prepared in the downstream of the KOTO detector. However, we found a detection gap in the edge of the in-beam photon counter just outside of the beam core. An acrylic Cherenkov counter named Beam Hole Guard Counter (BHGC) was developed as a photon-veto counter to cover the gap in the KOTO experiment.

The BHGC consists of a lead plate to convert photons, and an acrylic plate which acts as a Cherenkov radiator. One feature of the BHGC is neutron insensitiveness, which is important because there are large amount of neutrons scattered from the in-beam photon counter. Charged particles generated from neutrons tend to be slow and cannot make Cherenkov radiation when the velocity is below the Cherenkov threshold. The acrylic plate acts as a light guide to photomultipliers attached at the both ends of the acrylic plate and also helps to suppress neutron hits. Charged particles from neutrons may emit Cherenkov radiation if their speeds exceed the Cherenkov threshold. In this case, the Cherenkov angle tents to be smaller, and if the angle is smaller than the total reflection threshold in the acrylic plate, such photons cannot be transported to the edge of the acrylic plate.

The performance of the acrylic Cherenkov counter was studied with electron test beam, which was well reproduced with Monte Carlo (MC) simulation. The design of BHGC was optimized so that $80 \%$ of the gap-induced background can be reduced, and installed in the KOTO experimental area. The KOTO experiment took physics data with the BHGC in April and May 2015. The gain of photomultipliers of the BHGC is stable through the runs. We compared a light yield with that obtained with MC simulation and there was a discrepancy between data and MC simulations. The timing resolution was studied and achieved $0.37 \mathrm{~ns}$ from the sigma of a Gaussian fit.

Flavor Physics \& CP Violation 2015

May 25-29, 2015

Nagoya, Japan

* Speaker. 


\section{The KOTO experiment and in-beam photon detector}

The KOTO experiment at the Japan Proton Accelerater Research Complex (J-PARC) aims to observe the CP-violating rare decay, $K_{L} \rightarrow \pi^{0} v \bar{v}$. The branching ratio of this decay mode is suppressed in the Standard Model $\left(2.4 \times 10^{-11}\right)$ and its theoretical uncertainty is small (2\%) [1]. Therefore, this decay mode is sensitive to new physics beyond the Standard Model (SM) [2].

We identify the signal $K_{L} \rightarrow \pi^{0} v \bar{v}$ by detecting a single $\pi^{0}$ reconstructed from two photons, and requiring that there are no observable particles other than the two photons. In order to ensure it, we need a hermetic veto system with high detection efficiency (Figure 1). For the detection of particles escaping in the beam region, an in-beam photon counter was prepared in the downstream of the KOTO detector (Figure 2).

We found, however, a detection gap in the edge of the in-beam photon counter just outside of the beam core. This gap makes 11 background events at the SM level sensitivity for the $K_{L} \rightarrow \pi^{0} v \bar{v}$ search. In order to reduce them to less than 0.1 events, we need to prepare a new detector to cover the gap. There are large amount of neutrons around the beam core. Because of this, a new detector is required to have low neutron efficiency as well as high photon efficiency.

\section{Beam Hole Guard Counter (BHGC)}

\subsection{Principle of the acrylic Cherenkov detector}

We prepared a new photon-veto counter named Beam Hole Guard Counter (BHGC) to cover the gap. The BHGC consists of a lead plate to convert photons, and an acrylic plate which acts as a Cherenkov radiator. The BHGC was designed to be insensitive to neutrons with the following two steps.

The first step is a Cherenkov threshold. Charged particles generated from neutrons tend to be slow and cannot make Cherenkov radiation when the velocity is below the Cherenkov threshold.

The second is a total reflection threshold. The acrylic plate acts as a light guide to photomultiplier tubes (PMTs) attached at the both ends of the acrylic plate. Cherenkov radiation may be emitted if speed of charged particles generated from neutrons exceed the Cherenkov threshold. In this case, the Cherenkov angle tends to be smaller, and if the angle is smaller than the total reflection threshold in the acrylic plate, such photons cannot be transported to the edge of the acrylic plate (Figure 3).

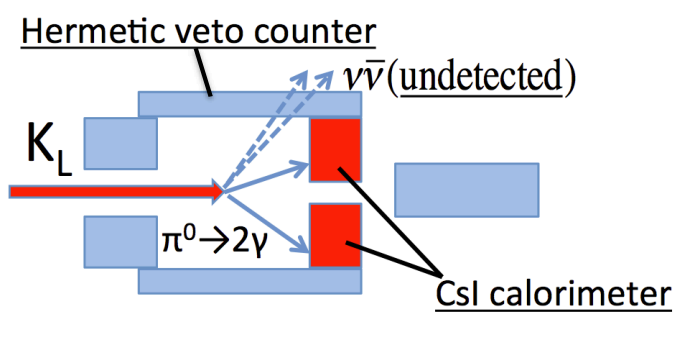

Figure 1: A schematic view of the KOTO detector system and a typical $K_{L} \rightarrow \pi^{0} v \bar{v}$ decay.

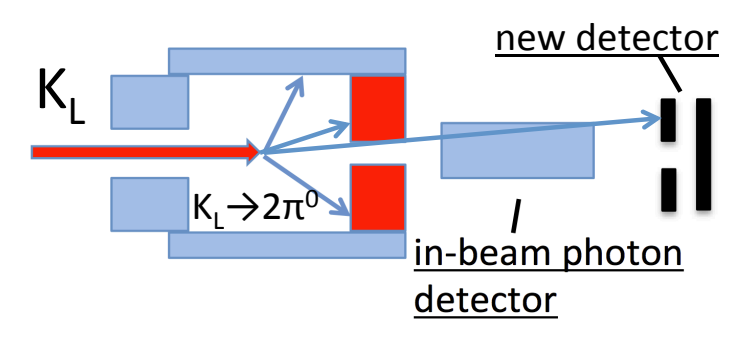

Figure 2: A typical $K_{L} \rightarrow 2 \pi^{0}$ background event to be detected by BHGC. 


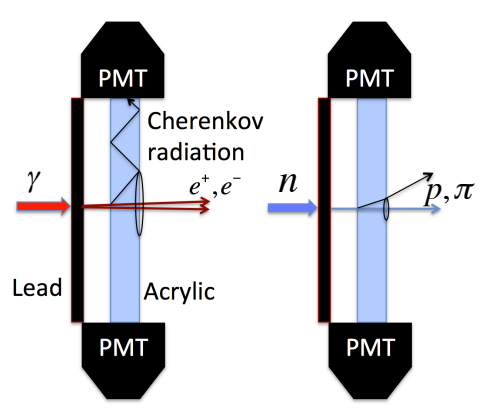

Figure 3: The principle of the BHGC. Cherenkov threshold: $\beta>0.67$. Total reflection threshold: $\beta>0.89$ (normal incident).

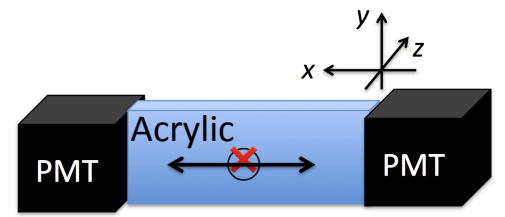

Figure 4: A schematic view of the prototype module for the beam test. The test beam direction is the same as the $z$ axis.

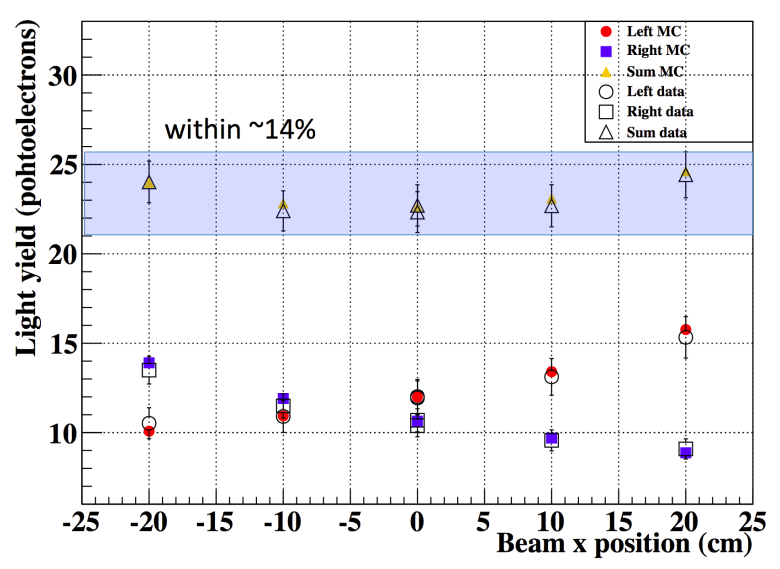

Figure 5: The position dependence of the light yields as a function of the beam $x$ positons.

\subsection{Test for light yield}

A test for light yield of the BHGC prototype module was performed using $500-\mathrm{MeV} / c$ electron beam at the Research Center for Electron Photon Science, at Tohoku University in September 2014. The prototype module consists of an acrylic plate and 5-inch PMTs which are coupled to both ends of the acrylic plate with optical grease. The acrylic plate is $500 \mathrm{~mm}$ wide, $120 \mathrm{~mm}$ high, and 10 mm thick (Figure 4).

The light yield of the acrylic Cherenkov counter for the prototype module was measured as a function of the incident beam position. Figure 5 shows the results of the data and a MC simulation. The uniformity of the light yields within $14 \%$ was achieved with the sum of the both-end readouts. The MC simulation including the ray tracing inside the acrylic reproduced the data well within the statistical errors.

\subsection{Design of the BHGC}

The thickness of the lead plate and the position of the BHGC was optimized with MC simulations, because these are related to the photon efficiency and the counting rate. 


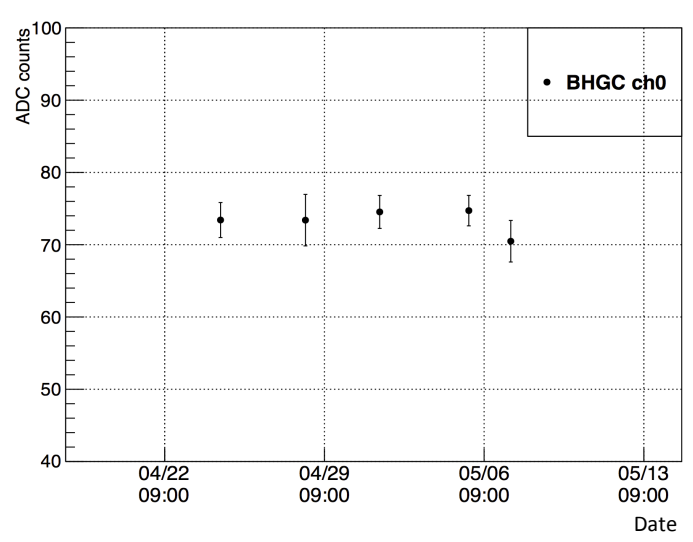

Figure 6: Gain stability through the runs.

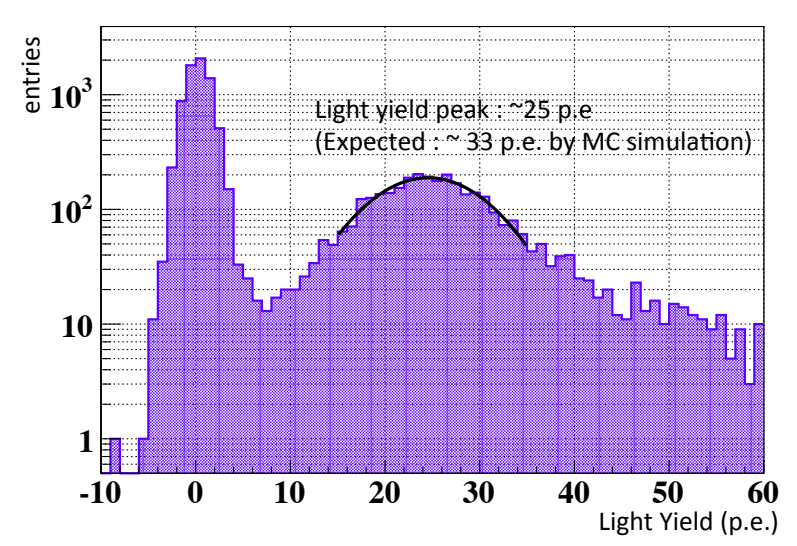

Figure 7: The number of photoelectrons distribution.

The thickness of the lead plate was determined to be $9.6 \mathrm{~mm}$. The position of the inner edge of the BHGC was determined to be $130 \mathrm{~mm}$ far from the beam axis. As a result, $80 \%$ of background events related to gap-going photons in the $K_{L} \rightarrow 2 \pi^{0}$ decay was estimated to be reduced in case that their energy is larger than $1 \mathrm{GeV}$. The single counting rate was also estimated to be $100 \mathrm{kHz}$, which is within a tolerable level for stable PMT operation.

After the design and construction, four BHGC modules were installed surrounding the beam at the most downstream of the KOTO experimental area in March 2015.

\section{Performance of the BHGC}

The KOTO experiment took physics data with the BHGC in April and May 2015. The data with BHGC was converted in the unit of the photoelectron using single photon information obtained from a light-emitting diode (LED) flushing trigger mixed in the physics runs. Figure 6 shows the stability of the single photoelectron gain of one BHGC channel for a part of the LED flushing triggers. Good stability was confirmed for all the channels through the period.

The light yield and timing resolution of each BHGC module were obtained using special runs, where upstream beam plug was closed to enhance high momentum muons or pions. In order to qualify the momentum, we further required hits of the in-beam photon detector, which consists of lead and aerogel sandwich with the Cherenkov threshold of 0.98 in $\beta$.

Figure 7 shows the light yield distribution of a BHGC module where the sum of the both ends was used. Because we used ultraviolet transparent acrylic for the actual BHGC module instead of normal acrylic for the case of the test beam, we expected the peak light yield was larger, and the expectation from MC simulation was 33 photoelectrons. However, we obtained the peak light yield of 25 photoelectrons from the special runs' data. We are investigating the reason of the discrepancy, although the stability of the light yield was confirmed with this method.

The timing resolution was studied using the timing difference of both ends as shown in Figure 8 , where hit position was constrained in the overlapping region of the BHGC modules. We obtained the resolution of $0.37 \mathrm{~ns}$ from the sigma of a Gaussian fit. It shows the BHGC module was operated as expected from the timing property obtained in the test beam. 


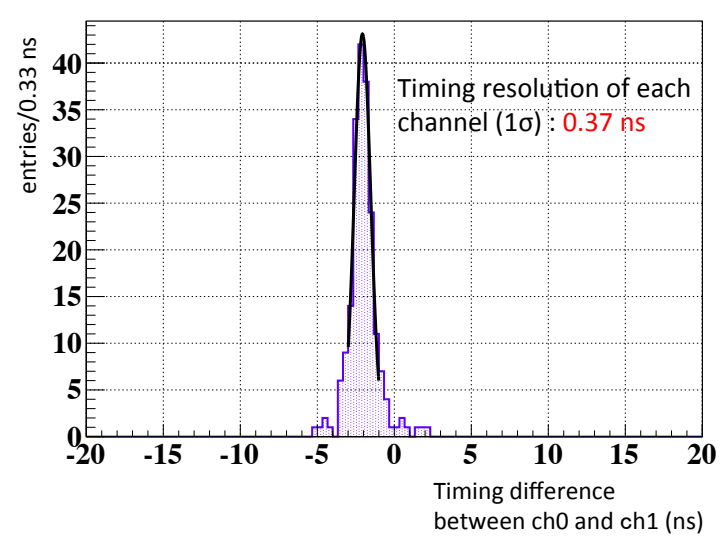

Figure 8: Timing difference distribution.

\section{Summary}

The acrylic Cherenkov counter named BHGC was developed for covering the detection gap. Using electron test beam, the performance of the acrylic Cherenkov counter was studied, which was well reproduced with MC simulations. The BHGC was designed so that $80 \%$ of the related backgrounds can be reduced, and installed in the KOTO experimental area. The basic performance of the BHGC in the KOTO physics run period was studied. We confirmed the stability of the PMTs with the single photoelectron information. Stability of the Cherenkov light yield and timing resolution were also studied by selecting high momentum charged particles. We will further investigate the discrepancy in the light yield between the data and $\mathrm{MC}$ in order to get more reliable estimate for the KOTO background reduction with the BHGC.

\section{References}

[1] J. Brod, M. Gorbahn and E. Stamou, Phys. Rev. D 83, 034030 (2011).

[2] F. Mescia and C. Smith, Phys. Rev. D76, 034017 (2007). 